\title{
A Spiking Neural Network for Illuminant-invariant Colour Discrimination
}

\author{
Sivalogeswaran Ratnasingam and Antonio Robles-Kelly
}

\begin{abstract}
In this paper, we propose a biologically inspired spiking neural network approach to obtaining an opponent pair which is invariant to illumination variations and can be employed for colour discrimination. The model is motivated by the neural mechanisms involved in processing the visual stimulus starting from the cone photo receptors to the centresurround receptive fields present in the retinal ganglion cells and the striate cortex. For our spiking neural network, we have employed the excitatory and inhibitory lateral synaptic connections, the Spike-Timing Dependent Plasticity (STDP) and long term potentiation and depression (LTP/LTD). Here, we employ a feed-forward leaky integrate-and-fire spiking neural network trained using a dataset of Munsell spectra. We have performed tests on perceptually similar colours under large illuminant power variations and done experiments on colourbased object recognition. We have also compared our results to those yielded by a number of alternatives.
\end{abstract}

\section{INTRODUCTION}

The human visual system has the remarkable ability of perceiving and discriminating object colours largely invariant to changes in illumination [8]. However, attempts to mimic the human visual system invariance to the illuminant have not been yet successful [16], [21]. In this paper, we investigate the possibility of achieving colour constancy via a biologically plausible spiking neural network which allows naturally for visual adaptation. From a bio-inspired perspective, the first chromatic visual adaptation model was proposed by von Kries based on the assumption that the sensitivity of the long, medium and short cone photoreceptors do not overlap each other [45]. Land and McCann [34] proposed a computational model known as "Retinex" for explaining the colour processing in the human visual system. Despite its success, the Retinex theory does not give a complete explanation of the human colour constancy [7]. Moreover, there is no widely accepted model that describes the colour constancy capability of the human visual system [21], [16].

This is despite the significant research effort in developing a biological model for human colour perception. Zmura and Lennie [15] have examined the light adaptation mechanism of the human visual system so as to achieve illuminant invariant colours. Ebner [17] proposed an approach based on the local space average of colour signals. Zickler et al. [46]

Sivalogeswaran Ratnasingam and Antonio Robles-Kelly are with National ICT Australia (NICTA), Locked Bag 8001, Canberra ACT 2601, Australia and the Research School of Eng., Australian National University, Canberra ACT 0200, Australia. NICTA is funded by the Australian Government as represented by the Department of Broadband, Communications and the Digital Economy and the Australian Research Council through the ICT Centre of Excellence program. (email: \{Siva.Ratnasingam, antonio.robleskelly\}@nicta.com.au).

This work was supported by NICTA introduced a colour space where the colour of an object can be represented approximately invariant with respect to the illuminant. Angeleopoulou [3] proposed a colour constancy algorithm based on the derivative spectra of the surface albedo by assuming Lambertian reflection. Buchsbaum [9] has proposed the Grey World algorithm, which is based on the assumption that the statistical mean colour of a scene is achromatic. This is somewhat similar to the work in [44], where the colour constancy problem is tackled assuming that the statistical mean of spatial derivative across the image is independent of the chroma. The colour constancy algorithms proposed by Finlayson and Drew [19] depart from the assumption that the illuminant power spectrum can be modelled using the black body radiators.

Note that the algorithms above employ approaches that do not, in general, model the neural processing involved in each layer of the visual path way. Along these lines, Dufort and Lumsden [14] have proposed a neural network model based upon the double opponent cells in the visual cortex. Moore et al. [35] have used neural networks to implement the Retinex algorithm. Courtney et al. [11] have used a multiple stage neural network for color constancy. Hérault [27] has used the logarithmic transduction of photoreceptors to justify the use of a low-pass filtering neural network for colour constancy.

In this paper, we propose a biologically inspired model for achieving illuminant invariant colour discrimination. We do this by modelling the short and long term synaptic plasticity rules found in the visual pathway. To this end, we model the double-opponent phenomenon per pixel using a spiking neural network. The topology of the network is biologically plausible, capturing the behaviour of excitatory and inhibitory lateral synaptic connections, short term learning rules and long term synaptic plasticity.

The paper is organised as follows. In Section II-A, we commence by ellaborating upon colour opponent signals and examine their formation inspired upon the centre-surround receptive fields. This motivates Section II-B, where we present our spiking neural network. We treat the relevant learning and synaptic plasticity processes in Section II-C. In Section III, we address implementation issues, such as encoding, parameter setup and training and provide some relevant discussion. Finally, in Sections IV and V, we present results and conclusions.

\section{COLOUR PROCESSING IN THE VISUAL SYSTEM}

In the human visual system, perception of different colours arises from three cone responses, namely long (L), medium (M) and short (S) in the retina. The first step in the visual 


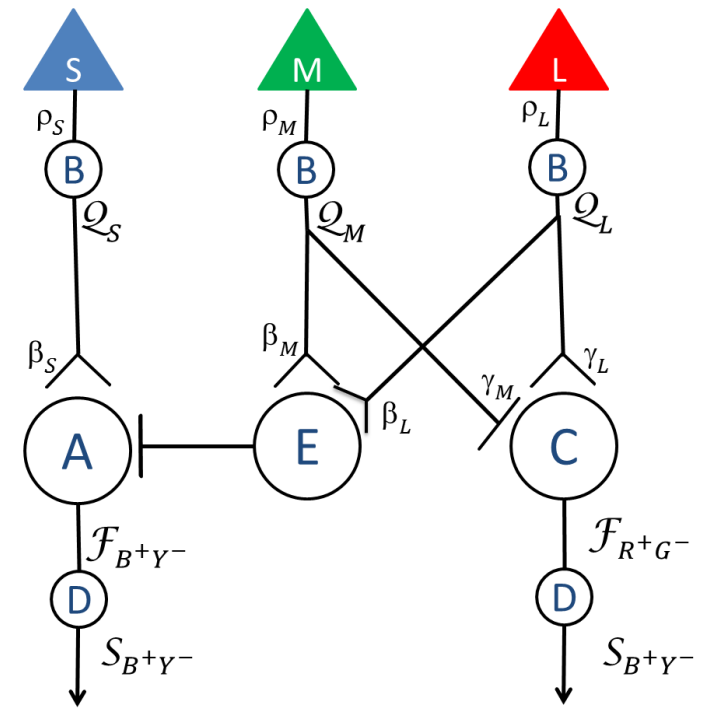

Fig. 1. Neural circuit showing how the two opponents (red-green and blueyellow) may be obtained using inputs from the long, medium and short (red, green and blue) cone responses.

processing is the light stimulation of photo receptors (cones and rods), which convert the photons into electrical potentials. These are converted into neural spikes in the centresurround receptive field of the retinal ganglion cells (RGN).

\section{A. Double Opponency}

The visual information conveyed by these neurons can be functionally classified into three categories, which are the red-green, blue-yellow opponent colour signals and an achromatic stimulus [12]. Furthermore, for a given value of the image radiance $I_{b}(u), b=\{L, M, S\}$, at pixel $u$ for either of the short $(\mathrm{S})$, medium $(\mathrm{M})$ or long $(\mathrm{L})$ cone responses, the colour visual perception can be mathematically characterised by $d \rho_{b}=\nu \frac{d I_{b}(u)}{I_{b}(u)}$, where $\nu$ is a constant which re-balances the colour response of the eye as the spectral composition of the scene changes, i.e. the adaptation condition [40]. This relation yields

$$
\rho_{b}=\nu \ln \left(I_{b}(u)\right)+\Upsilon
$$

where $\Upsilon$ is the so-called dark adaptation given by $-\nu \ln \left(\rho_{o}\right)$ and $\rho_{O}$ is the stimulus threshold of the photopic response.

Recall that the photoreceptors connect to the retinal horizontal cells, which, in turn, connect to the bipolar cells. Note that the bipolar cells can be viewed as neurons that, in a computational sense, encode the visual stimulus $\rho$ in such a manner that can be fed to the pre-synaptic potential of the corresponding retinal ganglion cells (RGC). This is an important observation since the RGCs exhibit properties which account for an opponent process over the blue-yellow $\left(B^{+} Y^{-}\right)$and red-green $\left(R^{+} G^{-}\right)$colour signals.

This opponent behaviour corresponds to the neural circuit in Figure 1. In the figure, the receptor stimulus is "encoded" by the $B$ neurons, which deliver a post-synaptic potential that can be then fed into the two opponent neurons, i.e. $A$ and
$C$. The neurons $A$ and $C$ deliver a train of spikes, which are "decoded" by the neurons D into two opponent "features" for colour discrimination.

In Section III, we discuss further the encoding and decoding neurons. For now, in the reminder of this section, we focus on the opponent cells. The pre-synaptic potentials of these two neurons are given by the weighted excitatory $(\langle)$ and inhibitory $(\mid)$ inputs for the short, medium and long signals which result in membrane potentials of the form

$$
\begin{aligned}
& \mathcal{G}_{R^{+} G^{-}}(t)=\gamma_{L} \mathcal{Q}_{L}(t)-\gamma_{M} \mathcal{Q}_{M}(t) \\
& \mathcal{G}_{B^{+} Y^{-}}(t)=\beta_{S} \mathcal{Q}_{S}(t)-\left(\beta_{L} \mathcal{Q}_{L}(t)+\beta_{M} \mathcal{Q}_{M}(t)\right)
\end{aligned}
$$

where $\gamma_{a}$ and $\beta_{b}$ with $a=\{M, S\}, b=\{L, M, S\}$ are constants that control the contribution of the long, medium and short colour stimuli.

\section{B. The Spiking Neural Network}

Note that, in the section above, we have treated the colour opponent signals in relation to the neural path way. In this section, we examine the formation of these two colour opponent signals inspired upon the centre-surround receptive fields. Recall that retinal ganglion cells fire action potentials in response to certain types of retinal stimulation. There are a number of neuron models that model these interactions. These include the Hodgkin-Huxley model [28], the Izhikevich model [29], the spike response model (SRM) [24] and leaky integrate-and-fire model (LIF) [26]. Amongst these, the LIF neuron provides a reasonably accurate neural behaviour which, from the computational viewpoint, does not require significant processing power and memory. In addition, training a leaky integrate-and-fire neuron is less complex than those corresponding to the Hodgkin-Huxley or Izhikevich models.

The proposed neural network is a feed forward one which consist of three layers. The number of neurons in each layer scales linearly with respect to the size of the input image. Each neuron in the network is modelled by LIF model, where the neuron "fires" a spike when the membrane potential crosses a threshold $\theta$. After firing, the neuron goes into a "refractory" period. During the refractory period the neuron does not fire any spikes. The integrate-and-fire neuron is, in effect, a resistor-capacitor (RC) circuit where the driving current $\mathcal{I}(t)$ at a time $t$ is given by

$$
\mathcal{I}(t)=\frac{u(t)}{R}+C \frac{d u(t)}{d t}
$$

where $u(t)$ is the membrane potential, $R$ is the resistor value and $C$ is the capacitance.

Rearranging the above equation results in

$$
\tau \frac{d u(t)}{d t}=-u(t)+R \mathcal{I}(t)
$$

where $\tau=R C$ is the time constant of the circuit, i.e. the membrane time constant of the neuron. Moreover, by definition, if the neuron fires at time $t_{i}$, then the membrane 
potential is reset to its "resting potential" $u_{r}$. This yields a membrane potential at time $t \geq t_{i}$ given by [26]

$u(t)=u_{r} \exp \left(-\frac{t-t_{i}}{\tau}\right)+\frac{1}{C} \int_{0}^{t-t_{i}} \exp \left(-\frac{s}{\tau}\right) \mathcal{G}_{h}(s) d s$

where $\mathcal{G}_{h}(t), h=\left\{R^{+} G^{-}, B^{+} Y^{-}\right\}$are the pre-synaptic potentials of the neurons at time $t \geq t_{i}$ as given in Equations 2 and 3.

With these membrane potentials, the post-synaptic spikes are, hence, given by

$$
\begin{aligned}
& \mathcal{F}_{R^{+} G^{-}}(t)=\delta\left(\mathcal{G}_{R^{+} G^{-}}(t) \geq \xi_{R^{+} G^{-}}\left(t_{i}\right)\right) \\
& \mathcal{F}_{B^{+} Y^{-}}(t)=\delta\left(\mathcal{G}_{B^{+} Y^{-}}(t) \geq \xi_{B^{+} Y^{-}}\left(t_{i}\right)\right)
\end{aligned}
$$

where $\delta(\cdot)$ is the delta function and $\xi_{R^{+} G^{-}}\left(t_{i}\right)$ and $\xi_{B^{+} Y^{-}}\left(t_{i}\right)$ are the firing thresholds for the opponent neurons.

\section{Learning and Synaptic Plasticity}

In the learning phase of a neural circuit, the synaptic plasticity between neurons and the neuronal excitability and dendritic integration are adapted so as to achieve a particular task, i.e. colour constancy, recognition, etc. For synaptic plasticity, Spike Timing Dependent Plasticity (STDP) [6] has been widely supported by recent biological experiments. STDP describes the post and pre-synaptic firing of the neurons in asymmetric form based upon the well known Hebbian rule [13], i.e. synapses increase their efficiency if they persistently excite the post-synaptic neuron.

In STDP, repeated pre-synaptic firing just before a postsynaptic action potential leads to long term potentiation (LTP) between the two neurons. This is as the repeated firing of the pre-synaptic spikes leads to long-term depression (LTD) of the synapse between the neurons. It is worth noting that, in the literature, there are a number of implementations of STDP. However, the nearest neighbour approach of the STDP has been supported by recent biological measurements [6], [38]. The nearest neighbour STDP (NN-STDP) takes into account only the nearest neighbour neuron pairs for adapting the synaptic plasticity. This contrasts with other classical STDP approaches, where all the pairs (pre and post-synaptic potentials) weigh equally in the adaptation of the synaptic plasticity. Moreover, this nearest neighbour implementation of the STDP is also compatible with the Bienenstock-CooperMunro (BCM) theory of learning found in the visual cortex [39].

The rationale underpinning NN-STDP hinges in the notion that, when the post-synaptic neuron fires a spike, it resets the membrane potential of the pre-synaptic neuron. Therefore, the most recent spike generated by the post-synaptic neuron overrides any effects of the earlier spikes. This is consistent with Equation 6, where we have defined the membrane potential as a function of the integration time, taken from the last spike time $t_{i}$.

For the long term adaptation in synaptic plasticity, we have used the weights $\gamma_{a}$ and $\beta_{b}$ in Equations 2 and 3. We follow
[25] and adapt these following the rule

$$
\omega\left(t_{i}\right)=\omega\left(t_{i-1}\right)+\triangle \omega
$$

where we have used $\omega\left(t_{i}\right)$ and $\omega\left(t_{i-1}\right)$ to denote the weight under consideration at the time of the current and previous spike and $\triangle \omega$ is the corresponding adaptation value.

Note that, once the post synaptic neuron has fired, subsequent spikes just after the current one are inhibited [38], [29]. This yields a Poisson process with respect to the threshold $\xi_{h}, h=\left\{R^{+} G^{-}, B^{+} Y^{-}\right\}$, where the post-synaptic spike density becomes exponential with respect to the time $t$, i.e. $\theta \exp ^{\theta_{h}\left(t_{i}\right) t}, \theta_{h}\left(t_{i}\right) \propto \xi_{h}\left(t_{i}\right)^{-1}$. If the threshold $\xi_{h}\left(t_{i}\right)$ is low, the firing rate is large and the timing interval between spikes is also small. This leads to potentiation whereas a low firing rate $\theta_{h}\left(t_{i}\right)$ leads to depression [37], [29].

This can be linked to the synaptic plasticity using the adaptation value $\triangle \omega$. We do this by using the potentiation $\phi_{p}$ and depression $\phi_{d}$ in [6], [37], [18]. This yields

$$
\begin{gathered}
\phi_{p}=\int_{0}^{\infty} \vartheta_{+} \exp ^{-\frac{t}{\tau_{+}}} \theta_{h}\left(t_{i}\right) \exp ^{-\theta_{h}\left(t_{i}\right) t} d t \\
\phi_{d}=\int_{-\infty}^{0} \vartheta_{-} \exp ^{\frac{t}{\tau-}} \theta_{h}\left(t_{i}\right) \exp ^{\theta_{h}\left(t_{i}\right) t} d t
\end{gathered}
$$

where $\tau_{-}$and $\tau_{+}$are time constants of the model at different phases and generally these values are taken to be $10 \mathrm{~ms}$ [37]. The parameters $\vartheta_{+}$and $\vartheta_{-}$depend on the current value of the synaptic plasticity, the adaptation value is given by the rule

$$
\Delta \omega= \begin{cases}\phi_{p} & \text { if } \theta_{h}\left(t_{i}\right)>\zeta \\ \phi_{d} & \text { if } \theta_{h}\left(t_{i}\right) \leq \zeta\end{cases}
$$

We also note that the effective magnitude of the synaptic plasticity can be set following [18]. This is

$$
\phi_{p, d}(\theta)=\theta_{h}\left(t_{i}\right)\left(\frac{\vartheta_{+} \tau_{+}}{1+\theta_{h}\left(t_{i}\right) \tau_{+}}+\frac{\vartheta_{-} \tau_{-}}{1+\theta_{h}\left(t_{i}\right) \tau_{-}}\right)
$$

Note that as high neural activity leads to synaptic potentiation, low neural activity leads to depression the value of threshold $\zeta$ that determines the transition between potentiation and depression. In [18], the authors show that this threshold can be expressed as

$$
\zeta=-\frac{\vartheta_{+} \tau_{+}+\vartheta_{-} \tau_{-}}{\tau_{+} \tau_{-}\left(\vartheta_{+}+\vartheta_{-}\right)}
$$

Moreover, lateral inhibition and excitation between neurons is often modelled by adapting the synaptic strength. However, here we consider the biological plausibility of the model and implement the lateral inhibition and excitation by adapting the spiking threshold $\xi_{h}, h=\left\{R^{+} G^{-}, B^{+} Y^{-}\right\}$. This is both, consistent with the equations above, where the synaptic plasticity is a function of $\theta_{h} \propto \xi_{h}^{-1}$ and experimental evidence showing that, in the cortical regions, neurons modify the firing threshold during the learning phase [10], [5]. As a result, we adapt the firing threshold according to the expression

$$
\theta_{h}\left(t_{i}\right)=\theta_{h}\left(t_{i-1}\right)+\Delta \theta_{h}
$$


where we have used $t_{i}$ and $t_{i-1}$ to denote the times of the current and previous spike, respectively.

To determine the adaptation value $\Delta \theta_{h}$ for the firing threshold, consider the two neurons $\mathrm{A}$ and $\mathrm{B}$ as given in Figure 1. If $\mathrm{A}$ and $\mathrm{B}$ fire at time $t_{i}$, both send an inhibition signal to each other. Similarly, if A fires and B does not then A inhibits neuron B while B excites A. This can be captured using the Poisson process mentioned earlier so as to define the adaptation at inhibition as

$$
\Delta \theta_{h}=-\exp ^{\left|\theta_{\text {ref }}-\theta_{h}\left(t_{i-1}\right)\right|}
$$

and at excitation as

$$
\Delta \theta_{h}=\exp ^{\left|\theta_{\text {ref }}-\theta_{h}\left(t_{i-1}\right)\right|}
$$

where $\theta_{\text {ref }}$ is the reference resting threshold.

\section{IMPLEMENTATION AND DISCUSSION}

\section{A. Encoding and Colour Descriptor Computation}

Note that, in the previous section we have not described in detail the structure of the encoding neurons B in Figure 1. Moreover, our aim of computation is two descriptors for colour discrimination which are approximately invariant to illuminant changes. To this end, we add two additional "decoders" at the output of neurons A and C. This is consistent with the notion that the stimuli $\rho_{b}$ has to be encoded and, at output, the spike train delivered by the neurons has to be decoded accordingly.

Moreover, note that, in Section II-A, we used the time $t$ as a variable which determines the behaviour of the network. As a result, encoding and decoding is a function of the time $t$ over a period $T$. Here, in order to reduce the computational complexity, we have used an encoding based upon a continuous pre-synaptic potentiation whose duration is determined by the normalised logarithmic stimulus corresponding to the image radiance. This is given by the rule

$$
\mathcal{Q}_{b}(t)= \begin{cases}1 & \text { if } t \leq T \frac{\rho_{b}}{\psi} \\ 0 & \text { Otherwise }\end{cases}
$$

where $\psi$ is a constant that corresponds to the maximum possible stimulus $\rho_{b}$ as given the dynamic range of the image.

For the decoders, we employ the sum of time differences for the $N$ spikes delivered over the corresponding period $T$. This yields

$$
\mathcal{S}_{h}=\sum_{i=1}^{N} t_{i}
$$

where, as before, $h=\left\{R^{+} G^{-}, B^{+} Y^{-}\right\}$and, $t_{i}$ is the relative time for the $i^{t h}$ spike.

\section{B. Parameter Setup}

Note that, in general, the behaviour of the spiking neurons is determined by a number of variables. These span from the time constants $\tau_{-}$and $\tau_{+}$to the threshold $\xi_{h}$. Moreover, the encoders also depend on the period $T$.

In Table I, we list the parameters used in the network and their settings. Note that these parameters are used to adapt the weights $\gamma_{a}$ and $\beta_{b}, a=\{M, S\}$ and $b=\{L, M, S\}$, in Equations 2 and 3 . These parameters are also used to adapt the spiking threshold and transition between potentiation and depression states for synaptic plasticity.

Here, we have set the dark adaptation to zero following the fact that this can be viewed as an offset in the colour stimulus. Similarly, we have set the rebalancing $\nu$ to unity without any loss of generality since this can be viewed as a constant that can be absorbed by the pre-synaptic weights. The encoding period $T$ and the potentiation and depression parameter $\vartheta_{+}$and $\vartheta_{-}$have been trained, whereas the other parameter values have been set based upon biological evidence [43], [37].

\section{Training}

To obtain the encoding period $T$ and the potentiation and depression parameters $\vartheta_{+}$and $\vartheta_{-}$, we have used a training phase based upon 202 Munsell reflectance spectra ${ }^{1}$ when illuminated by $6 \mathrm{CIE}$ standard illuminants representing common light sources.

Here, we take a learning approach based upon the Mahalanobis distance between nearest neighbours in the space spanned by $\mathcal{S}_{R^{+} G^{-}}$and $\mathcal{S}_{B^{+} Y^{-}}$. This is so as to find the parameters which best correspond to the illuminant invariance of the colour opponents. Thus, we recover the encoding period $T$ and the potentiation and depression parameter $\vartheta_{+}$ and $\vartheta_{-}$such that the difference in Mahalonobis distance between adjacent Munsell pairs is constant with respect to the standard light sources under consideration at unit illuminant power. We do this making use of a three-dimensional linear search over the space spanned by $T, \vartheta_{+}$and $\vartheta_{-}$.

Figure 2 shows the normalised space spanned by $\mathcal{S}_{R^{+} G^{-}}$ and $\mathcal{S}_{B^{+} Y^{-}}$. In the panel, each point shows the pseudo colour of the Munsell reflectance under a D65 illuminant rendered with the CIE standard colour matching functions [41]. Each cluster shows the features obtained when illuminated with the six standard illuminants. From Figure 2, it can be seen that

${ }^{1}$ Accessible at http://www.mmnt.net/db/0/0/cs.joensuu.fi/pub/
color/spectra/mspec
\begin{tabular}{|l|c|c|}
\hline Parameter & Symbol & Value \\
\hline Dark adaptation & $\Upsilon$ & 0 \\
Colour response rebalancing & $\nu$ & 1 \\
Capacitance & $C$ & $1.0 \mu \mathrm{F}$ \\
Resistance & $R$ & $10 \mathrm{M} \Omega$ \\
Time constant & $\tau$ & $10 \mathrm{~ms}$ \\
Resting potential & $u_{r}$ & $-65 \mathrm{mV}$ \\
Potentiation parameter & $\vartheta_{+}$ & 0.0001 \\
Depression parameter & $\vartheta_{-}$ & 0.0001 \\
Reference spiking threshold & $\theta_{r e f}^{-1}$ & $-55 \mathrm{mV}$ \\
Encoding period & $T$ & $700 \mathrm{~ms}$ \\
\hline
\end{tabular}

TABLE I

LIST OF PARAMETERS WITH THEIR CORRESPONDING SYMBOLS AND SETTINGS. 


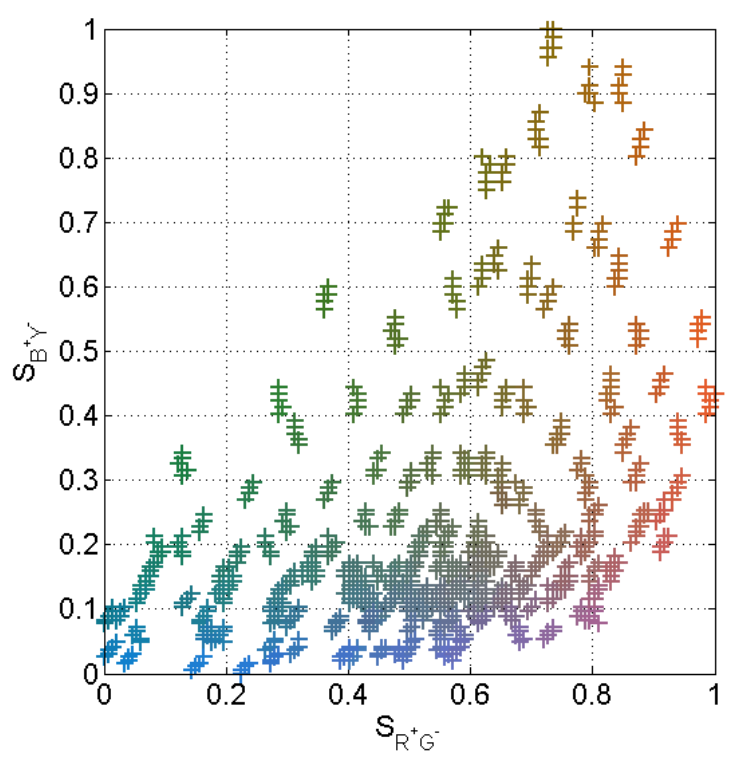

Fig. 2. Illuminant invariant colour opponent space formed by our proposed spiking neural network model. In this space illuminant invariant chromatic features obtained from some Munsell reflectance samples used in the 6 units test data set when illuminated by 6 spectra of the CIE standard illuminants. Each Munsell sample forms a small cluster when illuminated by different illuminants.

the colour varies smoothly across the space, with relatively compact clusters. This is an important observation since using either one of the decoded opponents can cause confusion between perceptually very different colours. However, using the two opponents together provides a good separation between Munsell pairs.

Moreover, our spiking neural network delivers a colour variation in this two-dimensional space which is similar to that of the CIE 1931 standard xy chromaticity space. This suggests that the output of our network exhibits a similar behaivour to that of the human visual system. In figure 3, the variation of perceptual distance in our chromaticity space is plotted against the CIELab distance. From these results it can be seen that the proposed chromaticity space is perceptually uniform.

\section{EXPERIMENTS}

In this section, we illustrate the ability of our network to recognise perceptually similar colours and perform colourbased object recognition. To do this, we have used three datasets. The first of these is the standard Munsell reflectance samples employed earlier. The second dataset used here comprises 2211 spectral reflectances taken from the Floral Reflectance Database [4] ${ }^{2}$. Finally, we have used the Simon Fraser University data set [23].

\section{A. Recognition of Perceptually Similar Colours}

We commence by recognising perceptually similar colours. Note that, in Figure 2, each of the Munsell samples form a

\footnotetext{
${ }^{2}$ The database is accessible at http://reflectance.co.uk/index.php
}

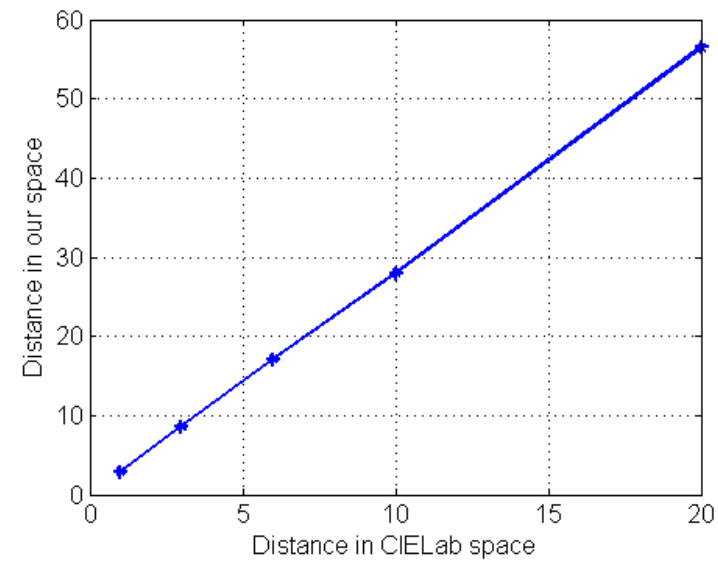

Fig. 3. The perceptual distance yielded by our opponents as a function of the CIELab colour distance.

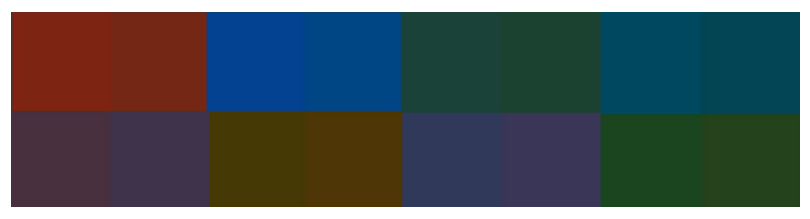

Fig. 4. Some of the Munsell reflectance samples used in the 6 units test data set. In each row two adjacent patches show the colour of the Munsell reflectance pairs in the data set when illuminated by D65 illuminant. The reflectances were normalised in such a way that the CIELab 'L' component is 50 units.

cluster when illuminated with the CIE standard illuminants under consideration. We commence by quantitatively assessing the degree of illuminant invariance and the ability of our network to recognise perceptually similar colours. To do this, we employ the standard Munsell reflectance samples, the Floral reflectance database [4] and a set of 20 light source spectra obtained from the CIE standard spectra ${ }^{3}$ with a temperature range of $\left[4000^{\circ} \mathrm{K}, 23000^{\circ} \mathrm{K}\right]$ in $1000^{\circ} \mathrm{K}$ intervals.

For both datasets, we have chosen two sets of test reflectance samples with 100 pairs in each set. The first of these sets is such that the pairs exhibit a pairwise distance of 6 units. The second set corresponds to pairs with a pairwise distance of 10 CIELab units. The reason for choosing these two (6 and 10) CIELab unit distances is based on the perceptual interpretation of the CIELab colour space. That is as 3 to 6 CIELab units are described as a good match and 6 to 10 CIELab units are an acceptable match of colour reproduction with respect to human colour perception [2]. Here we should also note that 3 to 5 units are often taken to be the just noticeable difference (JND) in technical and industrial applications [30]. Typical Munsell samples in the 6 unit Munsell reflectance test set are shown in Figure 4. In the figure, we show 8 pairs, where two adjacent patches on each row represent the reflectance pairs when illuminated by

\footnotetext{
${ }^{3}$ Accessible at http://mcsl.rit.edu/
} 


\begin{tabular}{|l|l|l|l|l|l|l|}
\hline & $\begin{array}{l}\text { CIELab } \\
\text { distance }\end{array}$ & $\begin{array}{l}\text { Our } \\
\text { method }\end{array}$ & $\begin{array}{l}\text { Finlayson and } \\
\text { Hordley[20] }\end{array}$ & $\begin{array}{l}\text { Gray } \\
\text { edge[31] }\end{array}$ & $\begin{array}{l}\text { Gray } \\
\text { world[9] }\end{array}$ & $\begin{array}{l}\text { White } \\
\text { patch[34] }\end{array}$ \\
\hline Munsell, & 6 & 56.6 & 32.7 & 40.6 & 40.8 & 40.5 \\
CIE light & 10 & 68.8 & 57.2 & 70.9 & 71.4 & 70.6 \\
\hline Floral, & 6 & 70.3 & 25.9 & 30.5 & 30.3 & 30.7 \\
CIE light & 10 & 87.0 & 49.6 & 48.0 & 47.9 & 47.6 \\
\hline
\end{tabular}

TABLE II

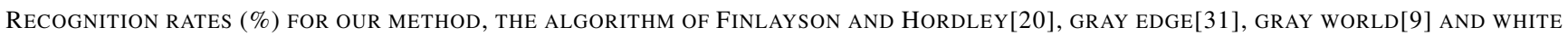
PATCH[34] ALGORITHMS WHEN TESTED WITH THE MUNSELL AND MEASURED FLORAL REFLECTANCES ILLUMINATED BY THE CIE STANDARD ILLUMINANTS IN [32].

\begin{tabular}{|l|l|l|}
\hline & $\begin{array}{l}\text { CIEDE2000 } \\
\text { distance }\end{array}$ & $\begin{array}{l}\text { Our method } \\
(\%)\end{array}$ \\
\hline Munsell, & 6 & 62.7 \\
CIE light & 10 & 73.0 \\
\hline Floral, & 6 & 82.8 \\
CIE light & 10 & 88.1 \\
\hline
\end{tabular}

TABLE III

TEST RESULTS FOR THE MUNSELL AND MEASURED FLORAL REFLECTANCE SETS WHEN ILLUMINATED WITH THE TWENTY CIE STANDARD ILLUMINANTS AS GENERATED USING THE CIEDE2000 COLOUR DIFFERENCE MODEL.

a D65 illuminant.

To assess the separability of the colours in our opponent space, we have used a nearest neighbour classifier based upon the Mahalanobis distance. In Table II, we compare the classification rates yielded by the classifier using our opponents $\mathcal{S}_{R^{+} G^{-}}$and $\mathcal{S}_{B^{+} Y^{-}}$against those delivered by the methods of Finlayson and Hordley [20] white patch algorithm [33], gray world algorithm [9] and gray edge algorithm [31]. From the table, note that our algorithm gives significantly better performance compared to the alternative. The reason is that our algorithm extracts two opponents whereas the Finlayson and Hordley [20] algorithm uses a single feature in which some perceptually different colours tend to "overlap". As the other three algorithms (white patch, gray world and gray edge) estimate a single illuminant for the entire scene and their non-realistic assumptions lead to degradation in performance in the real world scenes. Moreover, note that our network gives better performance on the Floral data set than on the Munsell samples. This is consistent with the observation that the human visual system is best suited to natural objects as compared to artificial colours [36].

We have also assessed the perceptual adaptation of our method by using the CIE standard colour difference model CIEDE2000 [1]. This model was developed so as to reflect the visual adaptation of the human visual system. In particular, it incorporates corrections for hue, lightness and chroma. We use this colour difference model to generate two sets of 100 pairs of reflectance spectra from the Munsell and the measured floral data sets. These reflectances were

\begin{tabular}{|l|l|l|l|}
\hline & $\begin{array}{l}\text { CIELab dis- } \\
\text { tance }\end{array}$ & $\begin{array}{l}\text { Lowest } \\
\text { luminance }\end{array}$ & $\begin{array}{l}\text { Highest } \\
\text { luminance }\end{array}$ \\
\hline Munsell & 6 & 56.6 & 55.5 \\
CIE light & 10 & 68.8 & 69.0 \\
\hline Floral & 6 & 70.3 & 70.3 \\
CIE light & 10 & 87.1 & 86.9 \\
\hline
\end{tabular}

TABLE IV

RECOGNITION RATES (\%) FOR THE MUNSELL AND FLORAL REFLECTANCE SPECTRA CORRESPONDING TO THE LOWEST AND HIGHEST LUMINANCE OVER A 6-DECADE VARIATION.

illuminated by the 20 CIE standard illuminants and employed for recognition of perceptually similar colours as described before. The recognition rates are shown in Table III. By comparing Tables II and III, we can conclude that our method performs better when applied to perceptually meaningful data. This suggests that our approach exhibits a behaviour akin to that of the human visual system in terms of perceptual adaptation and recognition of perceptually similar colours under different illuminants.

As the human visual system can recognise colours in scenes with large light variations, We now test our network by varying the dynamic range of the illuminants. To this end, we vary the illuminance of the 20 illuminants above in the order of 6-decades and rendered the spectra for both, the Munsell pairs used previously and the Floral Reflectance Database [4]. With the resultant colour pairs and floral reflectance spectra in hand, we have used a nearest neighbour classifier on the colours with the lowest and the highest luminance over this 6-decade range. The recognition rates are shown in Table IV. From these results we can conclude that our network can recognise perceptually similar colours under 6-decade luminance variation without a significant variation in performance.

\section{B. Colour Based Object Recognition}

We now test our opponents when used for illuminant invariant object recognition based upon colour. For this, we have used the bench marked Simon Fraser University data set [23]. This data set has been widely used by researchers for testing colour constancy algorithms. The data set is classified into four groups based on the light reflection 

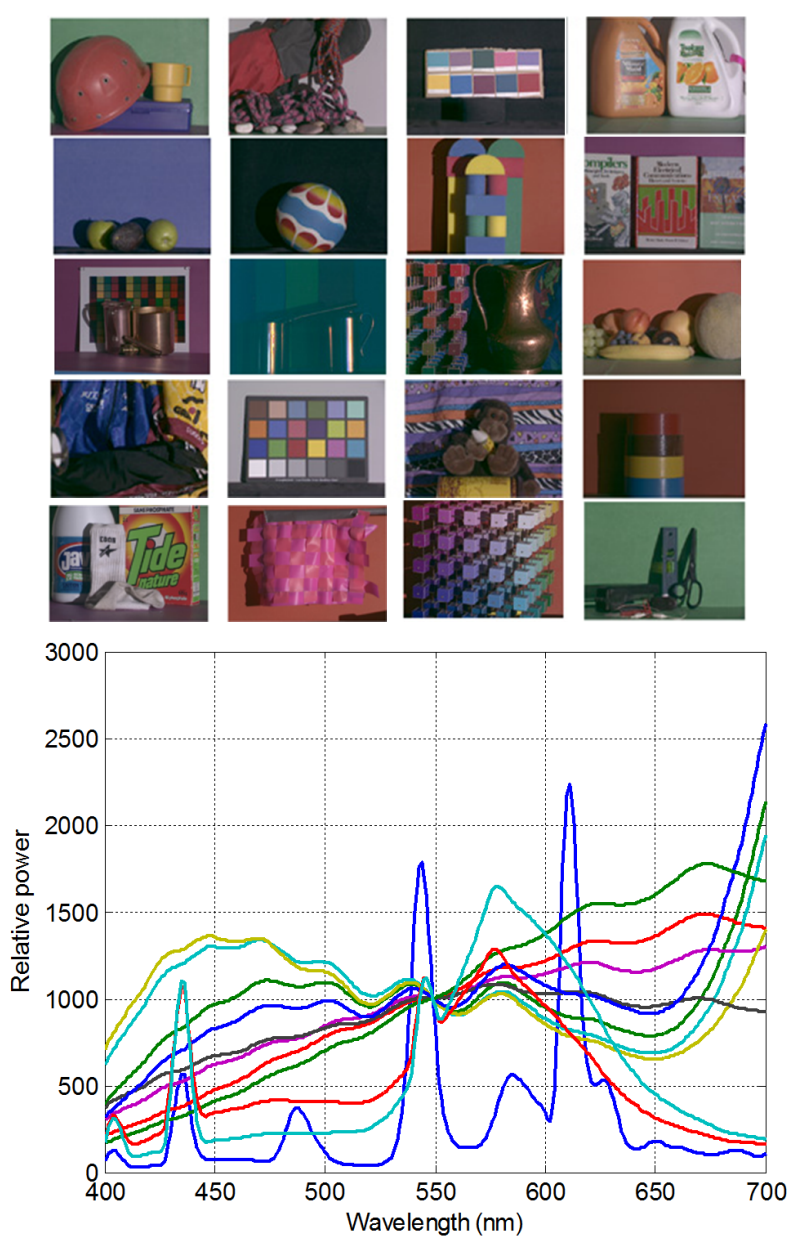

Fig. 5. Typical objects used in the experiment and spectral power distribution of the illuminants to illuminate the scenes

scenario. These are non-negligible dielectric specularities, metallic specularities, minimal specularities and at least one fluorescent surface. These images were captured by Sony DXC 930 camera when illuminated the scene with 11 different illuminants. These illuminants represent the common illuminants, daylight and fluorescent light sources. Typical images used in the experiment and the power spectra of the iluminants used to illuminate the objects are shown in Figure 5.

We have used the 429 images in the dataset and performed recognition based upon histogram intersection. Histogram intersection has been widely used for assessing the performance of colour constancy algorithms [42], [23], [22]. In the first step, illuminant invariant features are obtained for each image in the data set. These features are then used to construct a per-image histogram. Here, following on Funt et al. [23], we have used a two-dimensional histogram of $100 \times 100$ bins histogram for each image. The image of the query object is hence used to obtain a histogram and then intersected with those corresponding to the imagery in the data set. The image that yields the highest correlation with the histogram corresponding to the query object is declared as the best match.

For comparison, we have tested, alongside with our opponents, the colour constancy algorithms of Finlayson and Hordley [20], gray world [9], grey edge [31] and white patch [33]. Since histogram intersection can be viewed as a oneversus-all comparison method, we have effected experiments on two sets of imagery. The first of these corresponds to the full dataset, whereas the other one corresponds to a subset for each of the light reflection scenarios (we have excluded the fluorescent surfaces from this treatment as there are very few of these in the dataset). These subsets are randomly selected and experiments effected over 5 trials. We have done this in order to illustrate the effects of dataset size in performance. We have listed the recognition rates yielded by our opponents and the alternatives in Table V. From these results, we can conclude that our method gives better performance compared to all the other algorithms. Further, our network delivers opponents which give better results for both, matte and specular reflection.

\section{CONCLUSIONS}

In this paper we have proposed a spiking neural network whose layers represent various neurons in the visual pathway. Here, we have used a leaky integrate-and-fire neuron model and implemented the excitatory and inhibitory lateral connections, Spike-Timing Dependent Plasticity (STDP) and long term potentiation and depression (LTP/LTD) processes found in the visual pathway. We tested our model in terms of perceptual uniformity, discrimination of perceptually similar colours and colour-based object recognition under varying illumination conditions. We have also compared our results to those yielded by other methods.

Test results for colour and object recognition show that the proposed model outperforms the alternative methods. In particular, the performance of the model is comparable to that of the human visual system in recognising perceptually similar colours under varying illumination conditions. Therefore, this model could be used to understand the colour processing of the human visual system. In particular, it can provide a better understanding of how the human visual system perceives illuminant invariant colour of an object in the early stages of visual path way. It may also be of utility to understand visual processing in the cortical regions. In the future, we plan to improve the model by accommodating the neural processing involved in the extrastriate visual cortical areas.

\section{REFERENCES}

[1] The ciede2000 color-difference formula: Implementation notes, supplementary test data, and mathematical observations. Color Research \& Applications, 30(1):2130, 2005.

[2] A. Abrardo, V. Cappellini, M. Cappellini, and A. Mecocci. Artworks colour calibration using the vasari scanner. In Color Imaging Conference:Color Science,Systems and Applications, pages 94-97, 1996.

[3] E. Angeleopoulou. Objective colour from multispectral imaging. In In European Conf. on Computer Vision, pages 359-374, 2000.

[4] S. E. J. Arnold, V. Savolainen, and L. Chittka. The floral reflectance spectra database. In Nature Proceedings, page http://dx.doi.org/10.1038/npre.2008.1846.1., 2008. 


\begin{tabular}{|l|l|l|l|l|l|l|}
\hline Reflection type & $\begin{array}{l}\text { No of } \\
\text { objects }\end{array}$ & $\begin{array}{l}\text { Finlayson and } \\
\text { Hordley [20] }\end{array}$ & $\begin{array}{l}\text { White } \\
\text { patch [34] }\end{array}$ & $\begin{array}{l}\text { Grey } \\
\text { world [9] }\end{array}$ & $\begin{array}{l}\text { Grey } \\
\text { Edge [31] }\end{array}$ & $\begin{array}{l}\text { Our } \\
\text { method }\end{array}$ \\
\hline $\begin{array}{l}\text { Minimal } \\
\text { specularities }\end{array}$ & 5 & 66.0 & 88.0 & 84.0 & 22.0 & 94.5 \\
\cline { 2 - 7 } & All & 30.3 & 53.1 & 27.2 & 7.0 & 68.4 \\
\hline $\begin{array}{l}\text { Metallic } \\
\text { specularites }\end{array}$ & 5 & 74.5 & 78.2 & 54.5 & 30.9 & 94.5 \\
\cline { 2 - 7 } & All & 40.6 & 49.1 & 37.7 & 14.2 & 70.8 \\
\hline $\begin{array}{l}\text { Fluorescent } \\
\text { surfaces }\end{array}$ & All & 38.7 & 80.6 & 53.2 & 19.4 & 88.7 \\
\hline $\begin{array}{l}\text { Dielectric } \\
\text { specularities }\end{array}$ & 5 & 64.8 & 72.2 & 74.1 & 20.4 & 90.7 \\
\cline { 2 - 7 } & All & 36.7 & 45.9 & 47.9 & 11.2 & 69.4 \\
\hline
\end{tabular}

TABLE V

AVERAGE RECOGNITION RATES FOR THE OPPONENTS DELIVERED BY OUR NETWORK AND THE ALTERNATIVES.

[5] R. Azouz and C. M. Gray. Dynamic spike threshold reveals a mechanism for synaptic coincidence detection in cortical neurons in vivo. Proc. Natl. Acad. Sci. USA, 97:81108115, 2000.

[6] G. Bi and M. Poo. Synaptic modification of correlated activity: Hebb's postulate revisited. Ann. Rev. Neurosci, 24:139-166, 2001.

[7] D. Brainard and B. Wandell. Analysis of the retinex theory of color vision. J. Optical Society of America A, 3(10):1651-1661, 1986.

[8] D. H. Brainard, P. Longre, P. B. Delahunt, W. T. Freeman, J. M. Kraft, and B. Xiao. Bayesian model of human color constancy. Journal of Vision, 6:12671281, 2006.

[9] G. Buchsbaum. A spatial processor model for object colour perception. Journal of the Franklin Institute, 310(1):337-350, 1980.

[10] L. E. Chavez-Noriega, J. V. Halliwell, and T. V. Bliss. A decrease in firing threshold observed after induction of the epsp-spike (es) component of long-term potentiation in rat hippocampal slices. Experimental Brain Research, 79:633641, 1990.

[11] S. Courtney, L. Finkel, and G. Buchsbaum. Network simulations of retinal and cortical contributions to color constancy. Vision Research, 35(3):413-434, 1995.

[12] D. Dacey. Parallel pathways for spectral coding in primate retina. Annu. Rev. Neurosci, 23:743-775, 2000.

[13] H. D.O. The organization of behavior. New York: Wiley and Sons, 1949.

[14] P. A. Dufort and C. J. Lumsden. Color categorization and color constancy in a neural network model of v4. Biological Cybernetics, 65(4):293-303, 1991.

[15] M. D'Zmura and P. Lennie. Mechanisms of color constancy. J. Optical Society of America A, 3:1662-1672, 1986.

[16] M. Ebner. Colour constancy. IS\&T Series in Imaging Science and Technology, 2007.

[17] M. Ebner. How does the brain arrive at a color constant descriptor? In The 2nd international conference on Advances in brain vision and artificial intelligence, 2007.

[18] I. E.M and D. N.S. Relating stdp to bcm. Neural Computing, $15: 15111523,2003$.

[19] G. D. Finlayson and M. S. Drew. 4-sensor camera calibration for image representation invariant to shadingshadowslightingand specularities. In ICCV 2001, pages 473-480, 2001.

[20] G. D. Finlayson. and S. D. Hordley. Colour constancy at a pixel. J. Optical Society of America A, 18:253-264, 2001.

[21] D. H. Foster. Color constancy. Vision Research.

[22] B. Funt and G. Finlayson. Color constant color indexing. IEEE Trans. on Pattern Analysis and Machine Intelligence, 17(5):522-529, 1995.

[23] B.-K. Funt, B. and L. Martin. Is colour constancy good enough? In 5th European Conference on Computer Vision, pages 445-459, 1998.

[24] W. Gerstner. Time structure of the activity in neural network models. Physical Review E, 51:738758, 1995.

[25] W. Gerstner, R. Kempter, J. L. van Hemmen, and H. Wagner. A neuronal learning rule for sub-millisecond temporal coding. Nature, 386:76-78, 1996

[26] W. Gerstner and W. Kistler. Spiking neuron models : Single neurons, populations, plasticity. Cambridge University Press, 2002.

[27] J. Hérault. A model of colour processing in the retina of vertebrates: From photoreceptors to colour opposition and colour constancy phenomena. Neurocomputing, 12(2-3):113-129, 1996.

[28] A. Hodgkin and A. Huxley. A quantitative description of ion currents and its applications to conduction and excitation in nerve membranes.
J. of Physiology, 117:500544, 1952.

[29] E. Izhikevich. Simple model of spiking neurons. IEEE Trans. in Neural Networks, 14(6):15691572, 2003.

[30] J. J Hernandez-Andres, Raymond L. Lee and J. Romero. Color and luminance asymmetries in the clear sky. J. Appl. Opt, 42:458-464, 2003.

[31] T. G. Joost van de Weijer and A. Gijsenij. Edge-based color constancy. IEEE Transactions on Image Processing, 16(9):2207-2214, 2007.

[32] M. C. S. Laborartory. Daylight spectra. In http://mcsl.rit.edu/.

[33] E. Land. The retinex theory of color vision. Scientific American, 237(6):108-128, 1977.

[34] E. H. Land and J. J. McCann. Lightness and retinex theory. J. Optical Society of America A, 61:1-11, 1971.

[35] A. Moore, J. Allman, and R. M. Goodman. A real-time neural system for color constancy. IEEE Trans. Neural Networks, 2:237247, 1991.

[36] D. L. Ruderman, T. W. Cronin, and C. C. Chiao. Statistics of cone responses to natural images: implications for visual coding. J. Optical Society of America A, 15(8):20362045, 1998.

[37] P. J. Sjostrom, , and W. Gerstner. Spike-timing dependent plasticity. Scholarpedia, 5(3), 2010.

[38] P. J. Sjostrom, E. A. Rancz, A. Roth, and M. Hausser. Dendritic excitability and synaptic plasticity. Physiological Reviews, 88:769840, 2008.

[39] P. J. Sjostrom, G. G. Turrigiano, and S. B. Nelson. Rate, timing, and cooperativity jointly determine cortical synaptic plasticity. Neuron, 32:11491164, 2001.

[40] S. Stevens. Psychophysics: introduction to its perceptual, neural, and social prospects. Transaction Publishers, (ISBN 978-0-88738-643-5), 2007.

[41] W. S. Stiles and J. M. Burch. Interim report to the Commission Internationale de l'Éclairage Zurich, 1955, on the National Physical Laboratory's investigation of colour-matching. Optica Acta, 2:168$181,1955$.

[42] M. Swain and D. Ballard. Colour indexing. Int. J. Comp Vision, 7:11-32, 1991.

[43] T. P. Trappenberg. Fundamentals of computational neuroscience. Oxford University Press, 2010.

[44] J. van de Weijer, T. Gevers, and A. Gijsenij. Edge-based color constancy. IEEE Trans. Image Processing, 16(9):2207-2214, 2007.

[45] J. von Kries. Beitrag zur physiologie der gesichtsempfinding. Arch. Anat.Physiol, 2:5050-524, 1878.

[46] T. Zickler, S. P. Mallick, D. J. Kriegman, and P. N. Belhumeur. Color subspaces as photometric invariants. International Journal of Computer Vision, 79(1):13-30, 2008. 